\title{
A multiscale collocation method for fractional differential problems
}

\author{
L. Pezza, F. Pitolli* \\ Dip. SBAI, Università di Roma “La Sapienza”, Via A. Scarpa 16, 00161 Roma, Italy \\ Received 1 March 2016; received in revised form 4 July 2017; accepted 20 July 2017 \\ Available online 31 July 2017
}

\begin{abstract}
We introduce a multiscale collocation method to numerically solve differential problems involving both ordinary and fractional derivatives of high order. The proposed method uses multiresolution analyses (MRA) as approximating spaces and takes advantage of a finite difference formula that allows us to express both ordinary and fractional derivatives of the approximating function in a closed form. Thus, the method is easy to implement, accurate and efficient. The convergence and the stability of the multiscale collocation method are proved and some numerical results are shown.

(c) 2017 International Association for Mathematics and Computers in Simulation (IMACS). Published by Elsevier B.V. All rights reserved.
\end{abstract}

Keywords: Fractional differential problem; Collocation method; Fractional derivative; Fractional refinable function

\section{Introduction}

Fractional Calculus generalizes to positive real order the well-understood notion of derivative and integral of integer order (see, for instance, [19,24,26] and references therein). Even if the development of fractional calculus has a long history dating back to the 18th century, its use in real world applications has become popular just in more recent years. Indeed, in the last decades fractional differential problems are extensively used to model phenomena arising in several fields, from physics to continuum mechanics, from signal processing to electrochemistry, from biophysics to control theory. For instance, different kinds of fractional differential equations have been recently used to describe wave propagation in porous materials, diffusive phenomena in biological tissues, damping in viscoelastic mechanical systems, dynamic control of thermal systems. For a survey on applications see, for instance, $[12,13,17,25,27]$ and references therein. Along with the development of models there is a great effort to find out efficient numerical methods to solve fractional differential problems [2].

In this paper we introduce a multiscale collocation method to numerically solve fractional differential problems of order greater than 1. The method, which uses refinable functions as approximating functions, takes advantage of the refinability property to give rise to an accurate and efficient algorithm. Moreover, the fractional derivatives of the refinable functions appearing in the collocation matrix have a closed form that involves just the generalized

* Corresponding author.

E-mail addresses: laura.pezza@sbai.uniroma1.it (L. Pezza), francesca.pitolli@sbai.uniroma1.it (F. Pitolli). 
finite difference operator. All these reasons make the multiscale collocation method particularly attractive in solving fractional differential problems of different forms.

The paper is organized as follows. In Section 2 a general class of fractional differential problems recently used to model viscoelastic damping is described. The definition of fractional derivatives is also given. The multiscale collocation method is introduced in Section 3 where stability and convergence of the method are also proved. In Section 4 the analytical expression of both ordinary and fractional derivatives of refinable functions is given. Finally, in Section 5 the proposed method is used to numerically solve some fractional differential problems arising in continuum mechanics.

\section{Multi-term fractional differential equations}

Among the great variety of fractional differential problems used to model problem arising in real world applications, multi-term fractional differential equations have gained a great interest especially in the modeling of viscoelastic damping $[1,7,24]$. These problems are characterized by the presence of both ordinary and fractional derivatives, possibly of order greater than 1. In particular, in this paper we consider linear multi-term fractional differential equations of the form

$$
\left\{\begin{array}{l}
y^{(n)}(t)+\sum_{i=0}^{n_{0}} a_{i}(t) y^{(i)}(t)+\sum_{i=0}^{n_{1}} b_{i}(t)\left(D_{t}^{\gamma_{i}} y\right)(t)=f(t), \quad t \in[0, T], \\
y(0)=y^{\prime}(0)=\cdots=y^{(n-1)}(0)=0,
\end{array}\right.
$$

where

$$
\begin{aligned}
& n \in \mathbb{N}, \quad n_{0}, n_{1} \in \mathbb{N}_{0}:=\mathbb{N} \cup 0, \quad 0 \leq n_{0} \leq n-1, \\
& 0<\gamma_{0}<\cdots<\gamma_{n_{1}}<n, \quad \gamma_{i} \notin \mathbb{N}, \quad 0 \leq i \leq n_{1},
\end{aligned}
$$

and $a_{i}(t), 0 \leq i \leq n_{0}, b_{i}(t), 0 \leq i \leq n_{1}$, and $f(t)$ are some given continuous functions from $[0, T]$ into $\mathbb{R}$. Here, $D_{t}^{\gamma} y$ denotes the Caputo fractional derivative with respect to the time $t$ defined as

$$
D_{t}^{\gamma} y(t):=\left(\mathcal{J}^{(k-\gamma)} y^{(k)}\right)(t), \quad k-1<\gamma<k, \quad k \in \mathbb{N}, t>0
$$

where $\mathcal{J}^{(\gamma)}$ is the Riemann-Liouville integral operator

$$
\left(\mathcal{J}^{(\gamma)} y\right)(t):=\frac{1}{\Gamma(\gamma)} \int_{0}^{t} y(\tau)(t-\tau)^{\gamma-1} d \tau,
$$

and $\Gamma$ denotes the Euler's gamma function

$$
\Gamma(\gamma):=\int_{0}^{\infty} \tau^{\gamma-1} \mathrm{e}^{-\tau} d \tau
$$

We notice that, due to the homogeneous initial condition for the function $y(t)$, the Caputo definition (2.2) coincides with the Riemann-Liouville definition

$$
D_{t}^{\gamma} y(t):=\frac{d^{k}}{d t^{k}}\left(\mathcal{J}^{(\gamma)} y\right)(t), \quad t>0,
$$

and both reduce to the usual differential operator in case $\gamma \in \mathbb{N}[24,26]$. One of the advantage of the RiemannLiouville definition is in that the usual differentiation operator in the Fourier domain can be easily extended to the fractional case, i.e.,

$$
\mathcal{F}\left(D_{t}^{\gamma} y(t)\right)=(i \omega)^{\gamma} \mathcal{F}(y(t)), \quad \gamma \in \mathbb{R}^{+},
$$

where $\mathcal{F}(y)$ denotes the Fourier transform of the function $y$. Moreover, the Riemann-Liouville definition coincides with the Grunwald-Letnikov definition

$$
D_{t}^{\gamma} y(t)=\lim _{\delta \rightarrow 0} \frac{1}{\delta^{n}} \sum_{k=0}^{\frac{t}{\delta}}(-1)^{k}\left(\begin{array}{l}
\gamma \\
k
\end{array}\right) y(t-\delta k),
$$

where

$$
\left(\begin{array}{l}
\gamma \\
k
\end{array}\right):=\frac{\Gamma(\gamma+1)}{k ! \Gamma(\gamma-k+1)}, \quad k \in \mathbb{N}_{0}, \gamma \in \mathbb{R}^{+},
$$


are the generalized binomial coefficients. When $\gamma \in \mathbb{N}$, the generalized binomial coefficients reduce to the usual binomial coefficients so that the sequence $\left\{\left(\begin{array}{c}\gamma \\ k\end{array}\right)\right\}$ is compactly supported. When $\gamma \in \mathbb{R}^{+} \backslash \mathbb{N}$, the sequence is no longer compactly supported but the coefficients decay toward infinity as

$$
\left(\begin{array}{l}
\gamma \\
k
\end{array}\right) \rightarrow k^{-\gamma-1} \text { for } k \rightarrow \infty
$$

We notice that the Grunwald-Letnikov definition (2.7) is easier to use when addressing the numerical solution of fractional differential problems.

The multi-term fractional differential problem (2.1) was studied in [20] where the existence and uniqueness of its solution were proved. In that paper the authors proposed a spline collocation method to numerically solve (2.1). Multi-term fractional differential equations were studied also in [5] where they are solved by a multistep method (see also $[6,11,15]$ and references therein for different methods). In the next section we will introduce a new collocation method, the multiscale collocation method, which takes advantage of multiscale techniques to give rise to an efficient and accurate algorithm.

\section{The multiscale collocation method}

A sequence $\left\{V_{j}, j \in \mathbb{Z}\right\}$ of embedded approximating spaces, which are closed subspaces of $L^{2}(\mathbb{R})$, forms a multiresolution analysis (MRA) of $L^{2}(\mathbb{R})$ if
(i) $V_{j} \subset V_{j+1}, j \in \mathbb{Z}$
(ii) $\overline{\cup_{j \in \mathbb{Z}} V_{j}}=L^{2}(\mathbb{R})$;
(iii) $\bigcap_{j \in \mathbb{Z}} V_{j}=\{0\}$;
(iv) $f(t) \in V_{j} \leftrightarrow f(2 t) \in V_{j+1}, j \in \mathbb{Z}$;
(v) there exists a $L^{2}(\mathbb{R})$-stable basis in $V_{0}$.

A MRA can be generated by a refinable function, i.e., a function defined through a refinement mask $\mathbf{a}=\left\{a_{k} \in\right.$ $\mathbb{R}, k \in \mathbb{Z}\}$ and a refinement equation as follows,

$$
\varphi(t)=\sum_{k \in \mathbb{Z}} a_{k} \varphi(2 t-k), \quad t \in \mathbb{R} .
$$

Suitable conditions on the mask coefficients $\left\{a_{k}\right\}$ ensure the existence of a unique function $\varphi$ solution to (3.1), belonging to $L^{2}(\mathbb{R})$ and such that their integer translates $\{\varphi(t-k), k \in \mathbb{Z}\}$ form a $L^{2}\left(\mathbb{R}\right.$ )-stable basis in $V_{0}$ (see [18] for details). As a consequence, the refinable function $\varphi$ generates all the spaces $V_{j}$ through dilation and translation, i.e.,

$$
V_{j}=\overline{\operatorname{span}}\left\{\varphi\left(2^{j} t-k\right), k \in \mathbb{Z}\right\}, \quad j \in \mathbb{Z}, t \in \mathbb{R} .
$$

We say that a refinable function $\varphi$ has order of approximation $\alpha$ when

$$
\inf _{g \in V_{j}}\|f-g\|_{L_{2}(\mathbb{R})}=O\left(2^{-j \alpha}\right)
$$

for all $f \in \mathcal{H}^{\alpha}(\mathbb{R})($ the Sobolev space of order $\alpha)[18,29]$.

Now, let us denote by

$$
V_{j}^{0}[0, T]=\overline{\operatorname{span}}\left\{\varphi_{j k}(t), k \in \mathcal{N}_{j}\right\}, \quad j \geq j_{0}, t \in[0, T],
$$

the restriction of $V_{j}$ on the interval $[0, T]$ such that the basis functions $\varphi_{j k}$ fulfill the initial conditions

$$
\varphi_{j k}(0)=\varphi_{j k}^{\prime}(0)=\cdots=\varphi_{j k}^{(n-1)}(0)=0 .
$$

Here, $\mathcal{N}_{j} \subset \mathbb{Z}$ is the set of admissible index $k$ and $j_{0}$ is the initial multiresolution scale. Thus, $V_{j}^{0}[0, T]$ is generated by the $N_{j}$ functions $\varphi_{j k}$ with $N_{j}=\operatorname{dim}\left(\mathcal{N}_{j}\right)$.

For any $j$ held fix, the multiscale collocation method looks for an approximating function

$$
y_{j}(t)=\sum_{k \in \mathcal{N}_{j}} c_{j k} \varphi_{j k}(t) \in V_{j}^{0}[0, T]
$$

that solves the differential problem (2.1) on a set of collocation points. Due to (3.1), $\varphi_{j k}$ can be efficiently evaluated on dyadic nodes by the cascade algorithm [18], thus it seems natural to use dyadic nodes as collocation nodes. For 
any integer value $T$, let $\left\{t_{p}=p / 2^{s}, p=0, \ldots, T 2^{s}\right\}$, be a set of $N_{s}=T 2^{s}+1$ dyadic nodes in the interval $[0, T]$. Here, $s$ is a given non-negative integer such that $N_{s} \geq N_{j}$. Thus, the discretized version of (2.1) reads

$$
\left\{\begin{array}{l}
y_{j}^{(n)}\left(t_{p}\right)+\sum_{i=0}^{n_{0}} a_{i}\left(t_{p}\right) y_{j}^{(i)}\left(t_{p}\right)+\sum_{i=0}^{n_{1}} b_{i}\left(t_{p}\right)\left(D_{t}^{\gamma_{i}} y_{j}\right)\left(t_{p}\right)=f\left(t_{p}\right), \quad 0<p \leq T 2^{s}, \\
y_{j}(0)=y_{j}^{\prime}(0)=\cdots=y_{j}^{(n-1)}(0)=0 .
\end{array}\right.
$$

Using (3.6) in (3.7) and recalling (3.5), we get

$$
\sum_{k \in \mathcal{N}_{j}} c_{j k}\left(\varphi_{j k}^{(n)}\left(t_{p}\right)+\sum_{i=0}^{n_{0}} a_{i}\left(t_{p}\right) \varphi_{j k}^{(i)}\left(t_{p}\right)+\sum_{i=0}^{n_{1}} b_{i}\left(t_{p}\right)\left(D_{t}^{\gamma_{i}} \varphi_{j k}\right)\left(t_{p}\right)\right)=f\left(t_{p}\right), \quad 0<p \leq T 2^{s},
$$

where $\left\{c_{j k}, k \in \mathcal{N}_{j}\right\}$ are the unknown coefficients. The linear system (3.8) can be written in matrix form as

$$
A_{j} C_{j}=F, \quad A_{j}=A_{j 1}+A_{j 2}+A_{j 3},
$$

where

$$
\begin{aligned}
A_{j 1} & =\left(\varphi_{j k}^{(n)}\left(t_{p}\right)\right)_{0<p \leq 2^{s} T, k \in \mathcal{N}_{j}}, \\
A_{j 2} & =\left(\sum_{i=0}^{n_{0}} a_{i}\left(t_{p}\right) \varphi_{j k}^{(i)}\left(t_{p}\right)\right)_{0<p \leq 2^{s} T, k \in \mathcal{N}_{j}}, \\
A_{j 3} & =\left(\sum_{i=0}^{n_{1}} b_{i}\left(t_{p}\right) D_{t}^{\gamma_{i}} \varphi_{j k}\left(t_{p}\right)_{p k}\right)_{0<p \leq 2^{s} T, k \in \mathcal{N}_{j}}, \\
F & =\left(f\left(t_{p}\right)\right)_{0<p \leq 2^{s} T},
\end{aligned}
$$

and $C_{j}=\left(c_{j k}\right)_{k \in \mathcal{N}_{j}}$ is the unknown vector. Since the most celebrated refinable functions are compactly supported or have fast decay, in practical applications the collocation matrix $A_{j}$ is a band matrix with bandwidth equal to $2(|\operatorname{supp}(\varphi)|-1)$. When $2^{s} T=N_{j}$, the linear system (3.9) is square and the unknown vector $C_{j}$ can be recovered by a LU algorithm for band matrices. Since the basis functions $\varphi_{j k}$ and their derivatives are linearly independent, the collocation matrix $A_{j}$ is invertible. When $2^{s} T>N_{j}$, (3.9) is an overdetermined linear system that can be solved in the least squares sense [8].

We notice that the entries of $A_{j}$ involve the values of $\varphi_{j k}$ and its ordinary and fractional derivatives on the dyadic nodes $t_{p}$. As a consequence, $\varphi_{j k}\left(t_{p}\right)$ can be evaluated by usual refinement techniques [18] while $\varphi_{j k}^{(i)}\left(t_{p}\right)$ and $D_{t}^{\gamma_{i}} \varphi_{j k}\left(t_{p}\right)$ can be evaluated by finite difference formulas (see Section 4 ).

The stability and the convergence of the multiscale collocation method are proved in the following theorems.

Theorem 3.1. Let $\mathcal{H}^{s}[0, T]$ denote the Sobolev space equipped with the norm

$$
\|v\|_{s}=\left(|\widetilde{v}(0)|^{2}+\sum_{k \in \mathbb{Z} \backslash\{0\}}|k|^{2 s}|\widetilde{v}(k)|^{2}\right)^{\frac{1}{2}}, \quad v \in \mathcal{H}^{s}[0, T],
$$

where $\widetilde{v}(k)$ are the Fourier coefficients of $v$. Assume $y$ and $f$ in (2.1) belong to $\mathcal{H}^{s}[0, T]$ and $\mathcal{H}^{q-n}[0, T], 0 \leq s \leq q$, respectively. Moreover, assume $\varphi$ belongs to the Hölder space $\mathcal{C}^{d, r}(\mathbb{R})$ with $0 \leq s-n \leq d+r$.

Then, the multiscale collocation method (3.8) is stable, i.e., the inequality

$$
\left\|C_{j}\right\|_{\ell_{s}} \leq \eta\|F\|_{\ell_{s-n}}
$$

holds for any level $j$ with $\eta$ a constant independent of $j$.

Proof. The multi-term fractional differential equation (2.1) fulfills the hypotheses of the class of differential operators studied in [4]. Thus, the stability follows from [4, Proposition 6.4]. 
Theorem 3.2. Let $y, f$ and $\varphi$ satisfy the hypotheses of Theorem 3.1. Then, the multiscale collocation method (3.8) is convergent, i.e.,

$$
\left\|y-y_{j}\right\|_{s} \rightarrow 0 \text { as } j \rightarrow \infty .
$$

Moreover, the following error estimate holds

$$
\left\|y-y_{j}\right\|_{s} \leq \eta 2^{-j(q-s)}\|y\|_{q}, \quad 0 \leq s \leq q,
$$

where $\eta$ is a constant independent of $j$.

Proof. The convergence and the error estimate follow from Theorem 3.1 and [4, Theorem 6.3].

We observe that from the estimate (3.13) it follows that the order of accuracy of the method is $2^{-j(q-s)}$.

\section{Fractional derivatives of refinable functions}

Both ordinary and fractional derivatives of refinable functions can be expressed in the closed form by the generalized finite difference operator

$$
\Delta_{\delta}^{\gamma} v(t):=\frac{1}{\delta^{\gamma}} \sum_{k \in \mathbb{N}_{0}}(-1)^{k}\left(\begin{array}{l}
\gamma \\
k
\end{array}\right) v(t-\delta k), \quad \gamma \in \mathbb{R}^{+} .
$$

When $\gamma \in \mathbb{N},\left\{\left(\begin{array}{c}\gamma \\ k\end{array}\right)\right\}$ is a compactly supported sequence and $\Delta_{\delta}^{\gamma}$ reduces to the usual finite difference operator. When $\gamma \in \mathbb{R}^{+} \backslash \mathbb{N}$, the sequence $\left\{\left(\begin{array}{l}\gamma \\ k\end{array}\right)\right\}$ is absolutely summable (cf. (2.9)) so that the limit of the series (4.1) exists under suitable hypotheses on $v$.

Before to proceed with the differentiation rule for refinable functions we need the B-spline factorization theorem that involves the fractional B-splines, i.e., the B-splines of real order [28,29]. They are defined as

$$
B_{\alpha}(t):=\frac{\Delta_{\delta}^{\alpha+1} t_{+}^{\alpha}}{\Gamma(\alpha+1)}, \quad \alpha>-\frac{1}{2}
$$

where $t_{+}^{\alpha}:=\max \left(0, t^{\alpha}\right), t \geq 0$, is the fractional truncated power function. We notice that the fractional B-splines reduce to the well-known polynomial B-splines when $\alpha$ has an integer value.

Theorem 4.1. Let $\varphi$ be a refinable function generating a $M R A$ in $L^{2}(\mathbb{R})$ with approximation order $\alpha$. Then, $\varphi$ can be factorized as

$$
\varphi=B_{\alpha-1} * \varphi_{0},
$$

where $\alpha \geq 1$ and $\varphi_{0}$ is a distribution such that $\int \varphi_{0}=1$. Moreover, if $\varphi_{0} \in L^{2}(\mathbb{R})$, then $D_{t}^{\alpha} \varphi \in L_{2}(\mathbb{R})$ and the following differentiation rule holds

$$
D_{t}^{\gamma} \varphi=D_{t}^{\gamma}\left(B_{\alpha} * \varphi_{0}\right)=\Delta_{\delta}^{\gamma}\left(B_{\alpha-\gamma} * \varphi_{0}\right), \quad 0<\gamma \leq \alpha .
$$

Proof. The claim follows from some results in [29].

Now, we are in a position to prove the differentiation rule for $\varphi_{j k}$.

Corollary 4.2. Let $\varphi_{\alpha}=B_{\alpha} * \varphi_{0}$ be a refinable function with $\varphi_{0} \in L_{2}(\mathbb{R})$ and let $\varphi_{\alpha, j, k}(t):=\varphi_{\alpha}\left(2^{j} t-k\right)$. Then,

$$
D_{t}^{\gamma} \varphi_{\alpha, j, k}=\Delta_{2^{-j}}^{\gamma} \varphi_{\alpha-\gamma, j, k}, \quad 0<\gamma \leq \alpha .
$$

Proof. Let $\Phi_{\alpha}(t):=\varphi_{\alpha}\left(2^{j} t\right)$ so that $\varphi_{\alpha, j, k}(t)=\Phi_{\alpha}\left(t-2^{-j} k\right)=: \Phi_{\alpha, j, k}(t)$. Thus, the claim follows by applying (4.4) to the function $\Phi_{\alpha, j, k}(t)$.

Since any admissible refinable function belongs to $L^{2}(\mathbb{R})$, the series in (4.5) converges. Moreover, the generalized binomial coefficients decay like $k^{-\gamma-1}$ as $k \rightarrow+\infty$. Thus, for computational purposes $\Delta_{\delta}^{\gamma} \varphi_{\alpha-\gamma, j, k}$ can be approximated by a finite sum. 


\section{Numerical results}

In this section we will apply the multiscale collocation method described in the previous sections to solve a first order and a second order multi-term fractional differential problem. As approximating spaces we use the family of MRAs generated by the fractional refinable functions introduced in [21]. They can be defined through the convolution law

$$
\varphi_{\alpha, h}=B_{\alpha-2} * \varphi_{h}, \quad 2 \leq \alpha \leq h,
$$

where $\varphi_{h} \in L^{2}(\mathbb{R}) \cap C^{0}(\mathbb{R})$ is the elementary refinable function that generates all the refinable functions in the class. $\varphi_{h}$ is the solution to the refinement equation

$$
\varphi_{h}(t)=\sum_{k=0}^{3} a_{h, k} \varphi_{h}(2 t-k), \quad t \in \mathbb{R},
$$

with mask coefficients

$$
a_{h, 0}=a_{h, 3}=\frac{1}{2^{h}}, \quad a_{h, 1}=a_{h, 2}=1-\frac{1}{2^{h}} .
$$

Here, $h \geq \alpha$ is a real shape parameter that controls the shape of $\varphi_{\alpha, h}$. In particular, when $\alpha \in \mathbb{N}$ with $\alpha \geq 2$, $\varphi_{\alpha, h}$ is compactly supported, belongs to $C^{\alpha-2}(\mathbb{R})$ and reduces to the GP refinable functions introduced in [10]; in the particular case $h=\alpha \in \mathbb{N}_{0}, \varphi_{\alpha, \alpha} \in C^{\alpha-1}(\mathbb{R})$ coincides with the polynomial B-spline of degree $\alpha$. For any admissible value of the parameters $\alpha$ and $h, \varphi_{\alpha, h}$ belongs to $C^{\lfloor\alpha\rfloor-2}(\mathbb{R})$ and decays to the infinity rather rapidly so that it can be assumed compactly supported for computational reasons. As a consequence, $\varphi_{\alpha, h}$ can be efficiently evaluated on dyadic nodes by standard multiresolution techniques (cf. [18]). Finally, we notice that $\varphi_{\alpha, \alpha} \equiv B_{\alpha}$. (See [21] for further details and properties of the fractional refinable functions $\varphi_{\alpha, h}$.)

Now, let us denote by $V_{\alpha, h, j}^{0}[0, T]$ the multiresolution spaces generated by $\varphi_{\alpha, h}$ in the closed interval $[0, T]$, i.e.,

$$
V_{\alpha, h, j}^{0}[0, T]=\overline{\operatorname{span}}\left\{\varphi_{\alpha, h, j, k}(t), k \in \mathcal{N}_{j}\right\}, \quad j \geq j_{0} .
$$

We notice that the basis $\left\{\varphi_{\alpha, h, j, k}\right\}$ can be constructed by adapting to the fractional refinable functions the same techniques used in [9].

By Theorem 4.1 and [21, Theorem 5] the following differentiation rule holds

$$
D_{t}^{\gamma} \varphi_{\alpha, h}(t)=\Delta_{\delta}^{\gamma} \varphi_{\alpha-\gamma, h-\alpha+2}(t)=\frac{1}{\delta^{\gamma}} \sum_{k \in \mathbb{N}_{0}}(-1)^{k}\left(\begin{array}{l}
\alpha \\
k
\end{array}\right) \varphi_{\alpha-\gamma, h-\alpha+2}(t-\delta k), \quad 0<\gamma \leq \alpha,
$$

where the series converges for any admissible value of $\alpha$ and $h$.

\subsection{A first order fractional differential problem}

We consider the fractional differential problem of first order [14]

$$
\left\{\begin{array}{l}
y^{\prime}(t)+D_{t}^{0.8} y(t)+(1+t) y(t)=\frac{14}{\Gamma(3.8)} t^{1.8}+2.5 t^{2}+\frac{5}{\Gamma(3.8)}(1+t) t^{2.8}, \quad t \in[0,1], \\
y(0)=0 .
\end{array}\right.
$$

which can be seen as a multi-term fractional differential problem of type (2.1) with

$$
n=1, \quad n_{0}=0, \quad a_{0}(t)=1+t, \quad n_{1}=0, \quad \gamma_{0}=0, \quad b_{0}(t)=1 .
$$

Its exact solution is $y(t)=\frac{5}{\Gamma(3.8)} t^{2.8}$.

We numerically solve Eq. (5.6) by the multiscale collocation method with $s=8$ and $j=4$. As approximating functions we use four different refinable functions: the cubic B-spline $\varphi_{3,3} \equiv B_{3}$, the GP refinable function $\varphi_{3,3.8}$, the fractional refinable function $\varphi_{3.2,3.8}$ and the fractional B-spline $\varphi_{3.8,3.8} \equiv B_{3.8}$. The corresponding numerical solutions $y_{\alpha, h, 4}$ and errors $y-y_{\alpha, h, 4}$ are displayed in Fig. 1. In Fig. 2, the refinable function $\varphi_{3.8,3.8}$, its first derivative and its fractional derivative of order 0.8 are displayed along with the corresponding collocation matrix. 

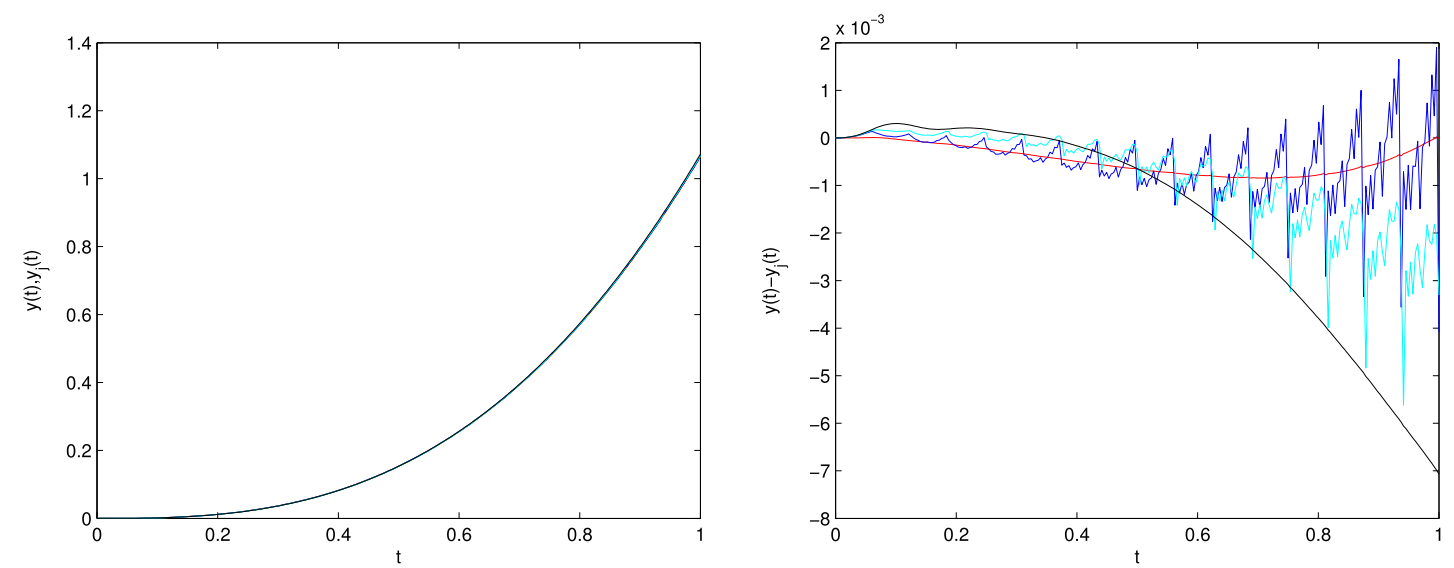

Fig. 1. Left panel: the graphs of the approximate solutions $y_{\alpha, h, 4}$ for $\alpha=h=3$ (red), $\alpha=3, h=3.8$ (blue), $\alpha=3.2, h=3.8$ (cyan) $\alpha=h=3.8$ (black) and of the exact solution $y(t)$, (green). Right panel: the graph of the error $y(t)-y_{\alpha, h, 4}(t)$. (For interpretation of the references to color in this figure legend, the reader is referred to the web version of this article.)
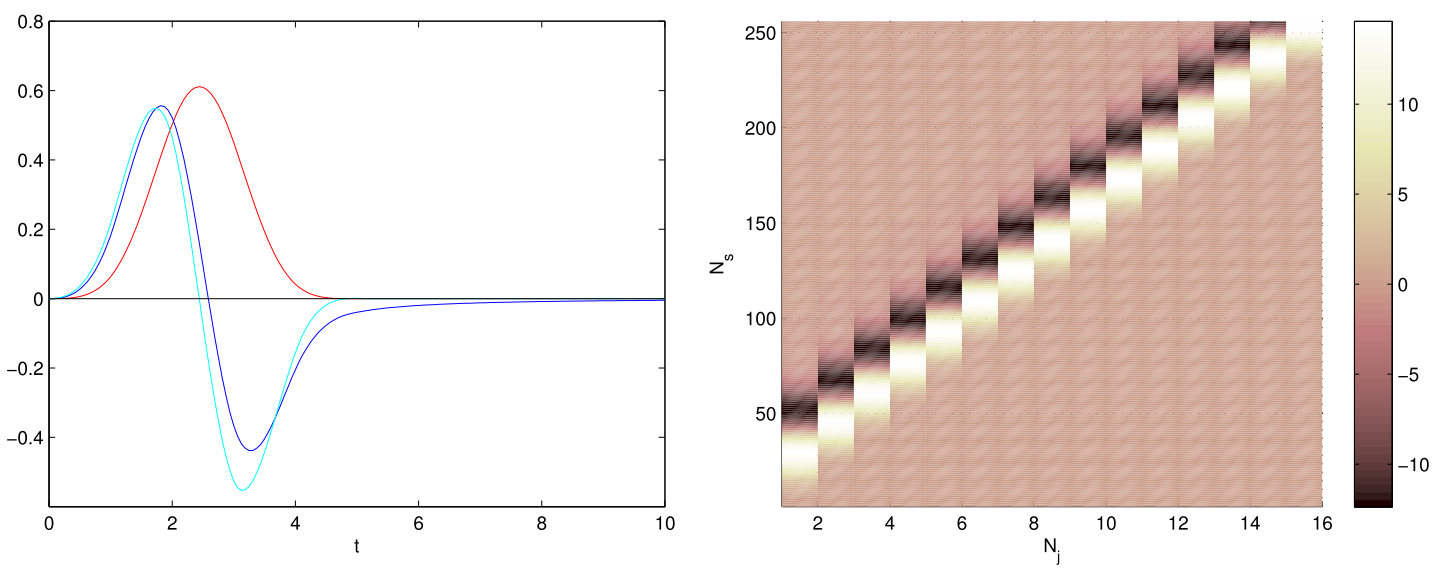

Fig. 2. Left panel: the graphs of $\varphi_{\alpha, h}$ (red), $\varphi_{\alpha, h}^{\prime}$ (cyan) and $\varphi_{\alpha, h}^{(0.8)}$ (blue) for $\alpha=h=3.8$. Right panel: the collocation matrix. (For interpretation of the references to color in this figure legend, the reader is referred to the web version of this article.)

Table 1

The error and the numerical rate of convergence when $\alpha=h=4$.

\begin{tabular}{lll}
\hline$j$ & $\left\|y-y_{4,4, j}\right\|_{0}$ & $R_{j}$ \\
\hline 4 & $1.22570 \mathrm{e}-3$ & \\
5 & $1.64860 \mathrm{e}-4$ & 2.81 \\
6 & $2.47992 \mathrm{e}-5$ & 2.82 \\
7 & $3.49323 \mathrm{e}-6$ & 2.83 \\
8 & $2.99257 \mathrm{e}-7$ & 2.81 \\
\hline
\end{tabular}

To check the convergence rate of the method we solved Eq. (5.6) when $s=10$ and for increasing values of $j$. Then, we evaluated the error $\left\|y-y_{\alpha, h, j}\right\|_{s}$ and the numerical rate of convergence defined as

$$
R_{j}=\log \left(\frac{\left\|y-y_{\alpha, h, j}\right\|_{s}}{\left\|y-y_{\alpha, h, j+1}\right\|_{s}}\right) \frac{1}{\log 2} .
$$

Table 1 shows the error $\left\|y-y_{\alpha, h, j}\right\|_{0}$ and the numerical convergence rate $R_{j}$ when $\alpha=h=4$. The numerical convergence rate is in good agreement with the theoretical rate which is equal to 2.8 . 

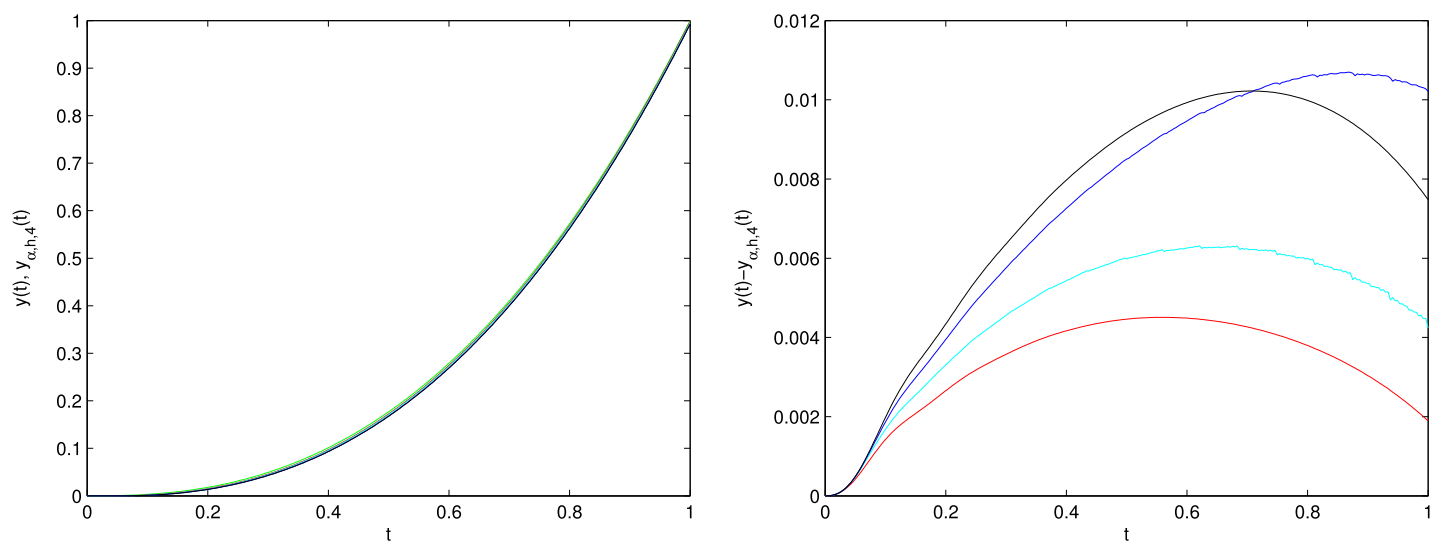

Fig. 3. Left panel: the graphs of the approximate solutions $y_{\alpha, h, 4}$ for $\alpha=h=4$ (red), $\alpha=4, h=4.5$ (cyan), $\alpha=4.2, h=4.5$ (blue) $\alpha=h=4.5$ (black) and of the exact solution $y(t)$ (green). Right panel: the graph of the error $y(t)-y_{\alpha, h, 4}(t)$. (For interpretation of the references to color in this figure legend, the reader is referred to the web version of this article.)
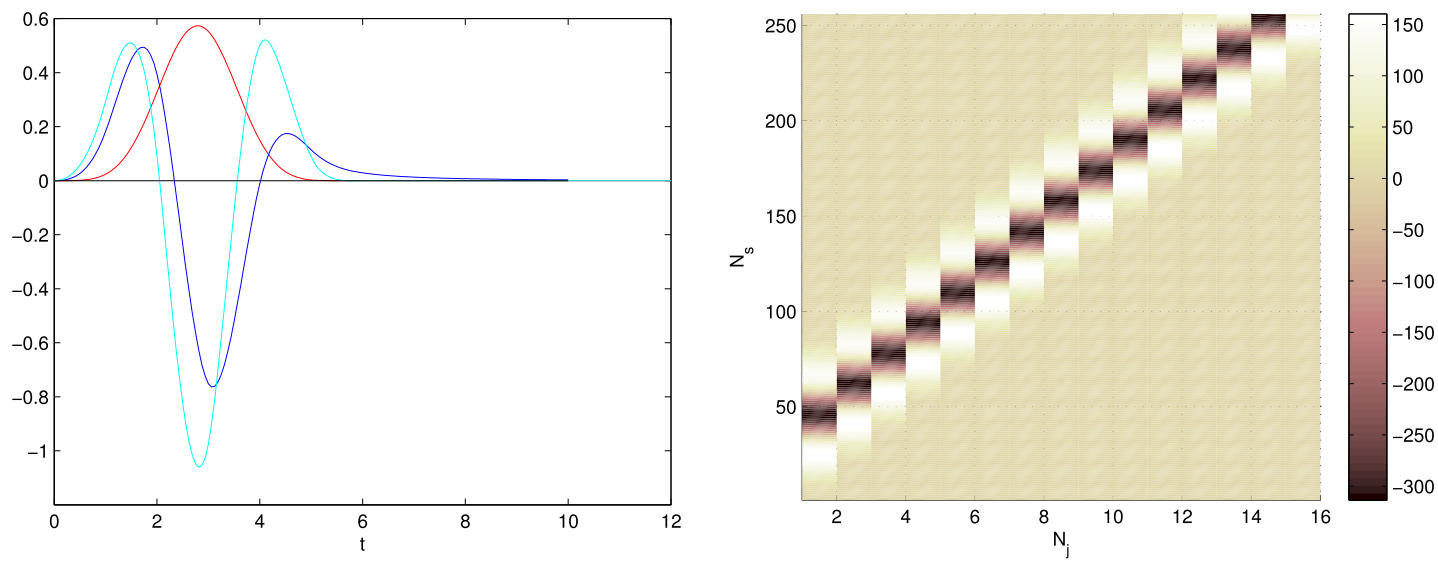

Fig. 4. Left panel: the graphs of $\varphi_{\alpha, h}$ (red), $\varphi_{\alpha, h}^{\prime \prime}$ (cyan) and $\varphi_{\alpha, h}^{(1.5)}$ (blue) for $\alpha=h=4.5$. Right panel: the collocation matrix. (For interpretation of the references to color in this figure legend, the reader is referred to the web version of this article.)

\subsection{A second order fractional differential problem}

We consider the Bagley-Torvik equation [1]

$$
\left\{\begin{array}{l}
y^{\prime \prime}(t)+D_{t}^{1.5} y(t)+y(t)=\frac{15}{4} \sqrt{t}+\frac{15}{8} \sqrt{\pi} t+t^{2} \sqrt{t}, \quad t \in[0,1] \\
y(0)=y^{\prime}(0)=0,
\end{array}\right.
$$

which is a second order multi-term fractional differential problem of type (2.1) with

$$
n=2, \quad n_{0}=0, \quad a_{0}(t)=1, \quad n_{1}=0, \quad \gamma_{0}=1.5, \quad b_{0}(t)=1 .
$$

Its exact solution is $y(t)=t^{2} \sqrt{t}[20]$.

We numerically solve Eq. (5.8) by the multiscale collocation method with $s=8$ and $j=4$. As approximating functions we use four different refinable functions: the quartic B-spline $\varphi_{4,4} \equiv B_{4}$, the GP refinable function $\varphi_{4,4.5}$, the fractional refinable function $\varphi_{4.2,4.5}$ and the fractional B-spline $\varphi_{4.5,4.5} \equiv B_{4.5}$. The corresponding numerical solutions $y_{\alpha, h, 4}$ and errors $y-y_{\alpha, h, 4}$ are displayed in Fig. 3. In Fig. 4, the refinable function $\varphi_{4.5,4.5}$, its second derivative and its fractional derivative of order 1.5 are displayed along with the corresponding collocation matrix. 
Table 2

The error and the numerical rate of convergence when $\alpha=h=5$.

\begin{tabular}{rll}
\hline \multicolumn{1}{c}{$j$} & $\left\|y-y_{5,5, j}\right\|_{1}$ & $R_{j}$ \\
\hline 6 & $4.45679 \mathrm{e}-2$ & \\
7 & $2.38904 \mathrm{e}-2$ & 0.90 \\
8 & $1.07152 \mathrm{e}-2$ & 1.17 \\
9 & $3.77161 \mathrm{e}-3$ & 1.51 \\
10 & $1.36391 \mathrm{e}-3$ & 1.48 \\
\hline
\end{tabular}

We check the convergence rate of the method also for Eq. (5.8). Table 2 shows the error $\left\|y-y_{\alpha, h, j}\right\|_{1}$ and the numerical convergence rate $R_{j}$ when $\alpha=h=5$. As $j$ increases, the numerical convergence rate tends to the theoretical rate which is equal to 1.5 .

\section{Conclusion}

We constructed a multiscale collocation method suitable to solve a class of multi-term fractional differential equations. We provided an explicit finite difference formula that allowed us to evaluate the fractional derivatives of the approximating function in an accurate and easy way. The method is proved to be stable and convergent and can be implemented efficiently by using standard multiscale techniques. Numerical results displayed in Section 5 show that the multiscale collocation method is able to approximate with high accuracy the solution of various fractional differential problems. We point out that the proposed method can be applied to solve other types of fractional differential problems, such as fractional diffusion equations (cf. [22]) or nonlinear fractional differential equations (cf. [23]). Moreover, the multiscale collocation method can be generalized to the case of non-uniform nodes using MRAs generated by refinable functions specially designed for this case (see, for instance, $[3,16])$.

\section{References}

[1] R.L. Bagley, P.J. Torvik, On the appearance of the fractional derivative in the behaviour of real materials, J. Appl. Mech. 51 (1984) $294-298$.

[2] D. Baleanu, K. Diethelm, E. Scalas, J.J. Trujillo, Fractional Calculus, Models and Numerical Methods, World Scientific, Singapore, 2012.

[3] M. Buhmann, C.A. Micchelli, Spline prewavelets for non-uniform knots, Numer. Math. 61 (1992) 455-474.

[4] W. Dahmen, S. Prössdorf, R. Schneider, Wavelet approximation methods for pseudodifferential equations: I Stability and convergence, Math. Z. 215 (1994) 583-620.

[5] K. Diethelm, N.J. Ford, Multi-order fractional differential equations and their numerical solution, Appl. Math. Comput. 154 (2004) 621-640.

[6] N.J. Ford, J.A. Connolly, Systems-based decomposition schemes for the approximate solution of multi-term fractional differential equations, J. Comput. Appl. Math. 229 (2009) 382-391.

[7] L. Gaul, P. Klein, S. Kempfle, Damping description involving fractional operators, Mech. Syst. Signal Process. 5 (1991) $81-88$.

[8] G.H. Golub, C.F. Van Loan, Matrix Computations, Johns Hopkins Univ Press, 1996.

[9] L. Gori, L. Pezza, F. Pitolli, Recent results on wavelet bases on the interval generated by GP refinable functions, Appl. Numer. Math. 51 (2004) 549-563.

[10] L. Gori, F. Pitolli, A class of totally positive refinable functions, Rend. Mat. Ser. VII 20 (2000) 305-322.

[11] X.-M. Gu, T.-Z. Huang, C.-C. Ji, B. Carpentieri, A.A. Alikhanov, Fast iterative method with a second order implicit difference scheme for time-space fractional convection-diffusion equations, J. Sci. Comput. (2017). http://dx.doi.org/10.1007/s10915-017-0388-9.

[12] R. Hilfer (Ed.), Applications of Fractional Calculus in Physics, World Scientific Publishing Co. Inc., River Edge, NJ, 2000.

[13] A.A. Kilbas, H.M. Srivastava, J.J. Trujillo, Theory and Applications of Fractional Differential Equations, in: North-Holland Mathematics Studies, vol. 204, Elsevier Science, Amsterdam, 2006.

[14] X. Li, Numerical solution of fractional differential equations using cubic B-spline wavelet collocation method, Commun. Nonlinear Sci. Numer. Simul. 17 (2012) 3934-3946.

[15] W.-H. Luo, T.-Z. Huang, G.-C. Wu, X.-M. Gu, Quadratic spline collocation method for the time fractional subdiffusion equation, Appl. Math. Comput. 276 (2016) 252-265.

[16] T. Lyche, K. Mørken, E. Quak, Theory and algorithms for non-uniform spline wavelets, in: N. Dyn, D. Leviatan, D. Levin, A. Pinkus (Eds.), Multivariate Approximation and Applications, Cambridge University Press, 2001, pp. 152-187.

[17] F. Mainardi, Fractional Calculus and Waves in Linear Viscoelasticity. An Introduction to Mathematical Models, Imperial College Press, London, 2010.

[18] S. Mallat, A Wavelet Tour of Signal Processing, Academic Press, 1999.

[19] K.B. Oldham, J. Spanier, The Fractional Calculus, Academic Press, 1974.

[20] A. Pedas, E. Tamme, On the convergence of spline collocation methods for solving fractional differential equations, J. Comput. Appl. Math. 235 (2011) 3502-3514.

[21] L. Pezza, Fractional GP refinable functions, Rend. Mat. Ser. VII 27 (2007) 73-87. 
[22] L. Pezza, F. Pitolli, A fractional spline collocation-Galerkin method for the fractional diffusion equation, (submitted for publication).

[23] F. Pitolli, L. Pezza, A fractional spline collocation method for the fractional order logistic equation, in: G. Fasshauer, L. Schumaker (Eds.), Approximation Theory XV: San Antonio 2016, in: Proceedings in Mathematics \& Statistics, vol. 201, Springer, 2017, pp. 307-318.

[24] I. Podlubny, Fractional Differential Equations, in: Mathematics in Science and Engineering, vol. 198, Academic Press, 1999.

[25] J. Sabatier, O.P. Agrawal, J.A. Machado, Advances in Fractional Calculus, Vol. 4, Springer, 2007.

[26] S.G. Samko, A.A. Kilbas, O.I. Marichev, Fractional Integrals and Derivatives: Theory and Applications, Gordon \& Breach, 1993.

[27] V.E. Tarasov, Fractional Dynamics. Applications of Fractional Calculus To Dynamics of Particles, Fields and Media, in: Nonlinear Physical Science, Springer, Heidelberg, 2010.

[28] M. Unser, T. Blu, Fractional splines and wavelets, SIAM Rev. 42 (2000) $43-67$.

[29] M. Unser, T. Blu, Wavelet theory demystified, IEEE Trans. Signal Process. 51 (2003) 470-483. 